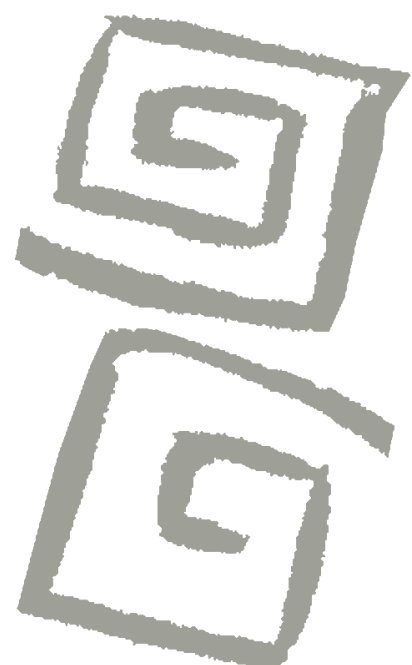

\title{
¿Sabemos lo que comemos?: Percepciones sobre el riesgo alimentario en Cataluña, España
}

\author{
Do we know what we eat?: Perceptions regarding food \\ risks in Catalonia, Spain
}

Eva Zafra Aparici' ${ }^{1}$ Araceli Muñoz García ${ }^{2}$, Cristina Larrea-Killinger ${ }^{3}$

'Doctora en Antropología Social. Profesora agregada interina, Universitat Rovira i Virgili, Tarragona, España. $\triangle$ iD

${ }^{2}$ Doctora en Antropología Social. Miembro del Observatorio de la Alimentación, Universidad de Barcelona, Barcelona, España. $\triangle$ iD

${ }^{3}$ Doctora en Antropología Social. Profesora titular; codirectora del Observatorio de la Alimentación, Universidad de Barcelona, Barcelona, España. $\bowtie$ (iD)
RESUMEN En este artículo se describen y analizan las percepciones sociales sobre el riesgo alimentario en Cataluña (España), que inciden en qué alimentos son percibidos como peligrosos, pero también en cómo, cuándo, dónde, por qué y de qué manera se concibe este riesgo a partir de las narrativas de los informantes. A través de un estudio cualitativo, se ha explorado cómo se construyen y gestionan los discursos legos, que conforman imaginarios diversos sobre el riesgo alimentario que no siempre coinciden con la mirada biomédica. Se señala que el riesgo sobre los alimentos no siempre se asocia a los peligros del progreso o la industrialización, ni se centra necesariamente en el debate dicotómico "alimento industrializado" versus "alimento natural", sino en un conjunto de posibilidades que tienen que ver también con el contexto de su producción, distribución, preparación y/o consumo.

PALABRAS CLAVES Alimentación; Riesgo; Percepción; Narrativas Personales; España.

ABSTRACT This article describes and analyzes social perceptions regarding food risks in Catalonia (Spain). In particular it uses the narratives of informants to determine which foods are perceived as dangerous and how, when, where and why this perception of risk develops. Through a qualitative research study, we explored how lay discourses are constructed and managed, creating diverse imaginaries regarding food risk that do not always coincide with the biomedical view. It is highlighted that food risk is not always associated with the dangers of progress or industrialization, nor is it necessarily focused on the dichotomous debate of "industrially produced food" versus "natural food"; rather food risk perceptions revolve around a series of possibilities that are also related to the production, distribution, preparation and/or consumption of food.

KEY WORDS Feeding; Risk; Perception; Personal Narratives; Spain. 


\section{INTRODUCCIÓN}

Las sustancias químicas utilizadas en la producción agrícola e industrial, junto con el aumento continuo de la oferta y la demanda de productos alimentarios diversos y de formas diferentes de producir, comer y consumir (funcionales, terapéuticas, ecológicas, locales y de proximidad, sostenibles, responsables, hedonistas, autónomas, solidarias...) comportan cambios en las percepciones sociales sobre el riesgo y la alimentación. En Cataluña, se ha observado que el índice de confianza en la alimentación ha bajado respecto a años anteriores en un 2,6\% en general $y$ en un $8,4 \%$ en los hogares ${ }^{(1)}$. Asimismo, muchos estudios ${ }^{(2,3,4,5,6,7,8,9,10)}$ confirman que, en Europa, ha aumentado la percepción negativa sobre las aplicaciones biotecnológicas en la industria alimentaria y la preocupación por la contaminación del agua, el pescado y el uso de productos químicos y pesticidas ${ }^{(1)}$.

La mayor parte de estos estudios, así como los informes de reconocidas instituciones nacionales e internacionales -como la Organización de las Naciones Unidas para la Alimentación y la Agricultura (FAO) y la Organización Mundial de la Salud (OMS), entre muchas otras- y la mayoría de las políticas alimentarias y de educación nutricional que se derivan de ellos, enfocan el análisis del riesgo sanitario de los alimentos (food safety) y de su percepción en la industrialización, sus aplicaciones tecnológicas y los aspectos de la organización económica y política de nuestra sociedad actual. Se centran básicamente en el análisis bioquímico y tecnológico de los alimentos, de la cadena alimentaria y, siguiendo muchas veces enfoques macroeconómicos como los de la sociedad del riesgo(11), suelen atribuir las causas de la percepción negativa sobre la alimentación a lo lejana que la población siente estos procesos, al hecho de no conocerlos y/o a la idea de no saber qué comemos ${ }^{(12)}$.

Las inquietudes de la población con relación a la alimentación no pueden reducirse, sin embargo, a una cuestión de estar o no a favor del progreso o de la industrialización ni, por extensión, al debate dualizado y unívoco que muchas veces se hace sobre el alimento artificial o industrializado en contra del considerado natural, ecológico o no manipulado industrialmente.

En este sentido, nuestro estudio partió de la idea de que las percepciones sobre el riesgo alimentario son multifactoriales y van más allá de marcos interpretativos que centran exclusivamente su mirada y el análisis del fenómeno en las causas de la industrialización y el progreso de las sociedades capitalistas. De hecho, ya en 1973, Douglas afirmaba que el estudio sobre las percepciones del riesgo debía considerar también los factores simbólico-culturales ${ }^{(13)}$. Además, la mayoría de los conceptos sobre el riesgo desarrollados desde las ciencias sociales en las dos últimas décadas comparten la idea de que las percepciones son fruto de complejos procesos sociales, política y culturalmente construidos $^{(14,15,16)}$. Asimismo, algunos de estos autores ${ }^{(16)}$ nos advierten que las perspectivas que únicamente tienen en cuenta los factores macroeconómicos a menudo señalan de forma insuficiente cómo los propios individuos construyen las percepciones sobre el riesgo.

Por lo tanto, nuestra investigación también partió de una concepción activa de los actores legos con respecto a la recepción de los conocimientos expertos y la construcción de ideas y discursos en torno al riesgo alimentario. Este enfoque dinámico e interactivo nos ha permitido ver que, junto con variables socioculturales como la edad, el género, la formación, la profesión o el hábitat, coexisten otras cuestiones que tienen que ver con las propias experiencias y vivencias de los sujetos y que plantean la necesidad de nuevas formas de aproximación al estudio y la intervención sobre el riesgo y la alimentación.

En este sentido, hemos observado que el cuerpo -individual, social y político ${ }^{(17)}-$ va ocupando, cada vez más, el centro de la producción del discurso y las prácticas alrededor del riesgo y la alimentación, de manera que las percepciones sobre el "alimento contaminado" no solo se construyen conforme la visión biomédica de un cuerpo físico, 
químico o biológico ${ }^{(18)}$ que se introduce en el interior de un alimento (como los aditivos, los pesticidas o las hormona, por ejemplo), sino también en función de la corporalidad individual, social y política que lo envuelve: experiencias y vivencias con relación al alimento y a quien lo produce, distribuye, prepara o consume; símbolos gustativos, olfativos, visuales, religiosos o morales histórica y culturalmente asociados a ellos; políticas y economías que deciden, legislan y regulan qué se considera como alimentos seguros/inseguros y a través de ello ejercen también su (bio)poder ${ }^{(19)}$ sobre los cuerpos individuales y sociales.

Por todas estas razones, el objetivo de este artículo es describir y analizar las percepciones sociales sobre el riesgo alimentario en Cataluña (España), poniendo el foco no solo en qué alimentos son concebidos como peligrosos, sino también en cómo, cuándo, dónde, por qué y de qué manera emergen estas percepciones alrededor de estos, sobre la base de los propios discursos y experiencias de los informantes. Los resultados que presentamos forman parte del proyecto interdisciplinario "Cuerpos tóxicos y etnoepidemiología sociocultural de la contaminación interna por compuestos tóxicos persistentes (CTP) en España", adscrito al Departamento de Antropología Social de la Universidad de Barcelona, dirigido por la Dra. Cristina Larrea Killinger y que contó con el apoyo y la financiación del Programa Nacional de Proyectos de Investigación Fundamental del Ministerio de Economía y Competitividad (CSO 2010/18661).

\section{MATERIAL Y MÉTODOS}

Se realizó un estudio cualitativo, basado en entrevistas individuales semiestructuradas, el cual se llevó a cabo en Cataluña (Comunidad Autónoma de España), durante el período comprendido entre 2011 y 2013. Se efectuaron 43 entrevistas a personas con diversa conexión con los contaminantes o tóxicos alimentarios: profesional (veterinarios/ as, agricultores, bomberos/as, carniceros/as, personal de limpieza, etc.); sociopolítica (ecologistas y personas con responsabilidades y/o cargos políticos); y personas consumidoras de productos ecológicos o seguidoras de otros tipos de alimentaciones alternativas.

El tipo de muestra fue no-probabilística y propositiva o intencional, según los parámetros específicos del estudio, con la intención de encontrar la máxima variación, heterogeneidad y significatividad de los diferentes sectores profesionales, así como de obtener una muestra equilibrada con una representación similar entre sexos, grupos de edad (a partir de 30 años), nivel formativo y ámbito geográfico (rural-urbano).

Las personas participantes en el estudio fueron informadas de los objetivos y métodos de la investigación, se obtuvo el consentimiento informado de cada una de ellas y se garantizó el anonimato y la confidencialidad de los datos durante todo el proceso.

Las entrevistas fueron grabadas en audio. Posteriormente se procedió a su transcripción literal y a su análisis para poder identificar temas y patrones y crear códigos, categorías y familias siguiendo estrategias de la teoría fundamentada ${ }^{(20,21)}$ a través del programa informático ATLAS.ti. Los datos fueron analizados con este software y estratificados por sexo, edad, nivel de estudios y lugar de procedencia. Luego se construyeron diagramas y mapas conceptuales para representar gráficamente las relaciones existentes entre los diferentes códigos o categorías.

Por último, cabe señalar que se han utilizado pseudónimos para mostrar los casos que sustentan los resultados presentados a continuación.

\section{PERCEPCIONES SOBRE EL RIESGO ALIMENTARIO}

Los resultados muestran una gran riqueza y diversidad de formas de ver y entender el riesgo alimentario. Desde discursos 
que centran la mirada en el propio alimento (qué) y en toda una gradación sobre su naturalidad y/o artificialidad, hasta construcciones sobre su seguridad sanitaria basadas en cómo son producidos y controlados los alimentos y según las relaciones entre dosis, acumulación y tiempo de exposición a estos (cuánto). Asimismo, se ha constatado que la distancia física y social de su producción, distribución y preparación (quién, para quién o dónde) también influye en los procesos de construcción de las percepciones alimentarias.

\section{¿El riesgo alimentario está en el qué?}

Muchos informantes relacionan el riesgo alimentario con los alimentos industrializados, en contraposición con los que consideran alimentos naturales. Se trata de categorías complejas de analizar porque implican diversas y heterogéneas maneras de concebir lo natural y lo transformado. A pesar de ello, en términos generales, la naturalidad de un alimento se vincula, a menudo, al hecho de no haber sido transformado o manipulado industrialmente en alguna de sus diferentes fases de producción, distribución, conservación y/o preparación culinaria. A su vez, la categoría natural se relaciona con otras como son lo rural frente lo urbano, lo tradicional frente lo moderno, lo cercano frente lo lejano o lo local frente lo global.

En este sentido, Luisa comenta los riesgos que, según su experiencia laboral como técnica en alimentación y agricultura, supone para la salud y el medio ambiente una agricultura industrializada por el hecho de utilizar productos químicos:

La agricultura industrial supone pesticidas, herbicidas, fungicidas, plaguicidas, etc. Muchos de ellos están más o menos comprobado que son malos para la salud. Por ejemplo, el glifosato era considerado un producto de muy bajo riesgo a nivel ambiental y ahora leo artículos científicos que dicen otra cosa. (Luisa, 35 años, Barcelona)
Por otra parte, Gerard, floricultor de Tarragona, vincula el riesgo alimentario con los alimentos prefabricados, por su supuesta artificialidad y el desconocimiento de su procedencia. De la misma forma, Gerard asocia la naturalidad de un alimento a la cercanía de su producción:

La comida prefabricada es una cosa a la que le tengo mucha manía [...] por las grasas y cosas que llevan artificiales. Nosotros en casa somos mucho de lo natural, un producto cercano que más o menos sabes de dónde viene y que está bien. (Gerard, 66 años, Tarragona)

Además, Gerard cree que existen diferencias objetivables entre un alimento artificial (procesado industrialmente) y uno natural, ya que cambia el gusto, el olor, el color o la textura. Lo afirma mientras rememora las prácticas de su abuelo cuando él aún era un niño:

Los pollos de ahora no tienen el mismo gusto que tenía aquel pollo que los abuelos criaban al aire libre. Y los huevos, janda que no cambian! Cuando hablamos de industrial, comoporejemplo la fruta, pues se tiene que recoger verde y eso también hace que pierda mucho su sabor. Si un melocotón tiene que estar tres días en el supermercado y tiene que aguantar, pues no puede llegar maduro. Por lo tanto, lo tienes que recoger verde y tiene otro sabor. Un melocotón verde es agrio, no tiene tanto gusto. (Gerard, 66 años, Tarragona)

Este relato demuestra que las experiencias vividas, con relación a las características organolépticas de los alimentos, transmiten significados sobre las maneras en que un alimento ha sido producido y, por extensión, sobre su seguridad sanitaria. Además, dependiendo de si un alimento conserva el color o lo cambia, tiene un "exceso" o "defecto" respecto a los patrones y criterios de naturalidad establecidos, es percibido como más o menos tóxico, contaminante, seguro o inseguro para la salud $d^{(22,23,24)}$. 
Para Gerard, un alimento industrial no puede ser nunca como uno natural. Sobre todo, ha sido en su contexto familiar donde Gerard ha aprehendido la mayoría de sus conocimientos sobre agricultura y donde ha conformado las ideas dicotómicas entre alimentación de hoy y alimentación del pasado, entre alimento moderno y alimento tradicional y finalmente entre alimento industrial y alimento natural. En esta categorización contrapuesta, Gerard siempre concibe la segunda categoría como la más natural y segura. De hecho, algunas investigaciones señalan que, en Europa y América, la naturalidad de los alimentos va ligada a ideas comunes como biológico, saludable, plantas y medioambiente ${ }^{(25)}$. Además, parece ser que las personas que están a favor de los productos naturales continúan prefiriendo un producto natural aunque se demuestren los beneficios del artificial(26).

Por otra parte, encontramos discursos en los que la idea de natural va ligada a la de alimento ecológico. Sin embargo, también se ha demostrado que no todos los informantes están de acuerdo con que un alimento ecológico sea necesariamente un alimento seguro para la salud y libre de contaminantes químicos. De hecho, Raúl se cuestiona la naturalidad de la producción ecológica, así como la eficacia, a partir de su propia experiencia de más de quince años como veterinario:

Muchas veces lo de ecológico es un malentendido, es una percepción. Porque aquel conejo que crees que por haberse criado en el campo es ecológico, resulta que está medicado. Pero como lo produce la señora María, en el patio de su casa, pues parece que sea natural. Pero igual ha estado medicado sin control. O igual un cultivo ecológico está al lado de un transgénico... Eso de ecológico es muy difícil de controlar. (Raúl, 38 años, Tarragona)

Rosa, maestra de educación primaria, piensa que un alimento de producción supuestamente natural puede resultar mucho más peligroso que otro que haya estado bajo el control industrial. Precisamente, lo que a ella le preocupa es hasta qué punto el primero ha estado controlado industrialmente. Rosa ha ido construyendo esta idea a lo largo de los años y a partir de las experiencias vividas con su abuelo, tío y otros campesinos de su pueblo:

\begin{abstract}
$A$ veces puede que compremos alimentos en un mercado de un pueblo pensando que son naturales, que han estado cultivados en un huerto, de manera natural... y resulta que las aguas que han regado ese huerto están contaminadas, el campesino fumiga $y$ pone insecticidas sin control... y resulta que, en realidad, es más peligroso este alimento que el otro. Esto se puede ver en mi pueblo. (Rosa, 35 años, Castellón)
\end{abstract}

De igual manera, Julia pone en duda la naturalidad de los alimentos cuando afirma que:

...no hay nada, hoy en día, que sea natural. Estoy segura de que 100\% biológico no hay nada. (Julia, 63 años, Barcelona)

Sobre este tipo de posicionamientos alrededor de la naturalidad y los efectos en la salud de los alimentos ecológicos, también existen controversias científicas. Estudios recientes $^{(27)}$ afirman los beneficios de los cultivos y alimentos ecológicos con relación al gran contenido en antioxidantes y menos cantidad en cadmio y residuos de pesticidas respecto de los alimentos convencionales. Por el contrario, otros autores ${ }^{(28)}$ recuerdan que las alertas sanitarias en producción ecológica son mayores y que la normativa que se aplica a los productos ecológicos permite, en ocasiones, prácticas incluso perjudiciales para el medio ambiente. Asimismo, un estudio reciente sobre la comida ecológica en la ciudad de Barcelona (España) evidencia la multitud de contradicciones y paradojas que afloran de los discursos sociales en torno a este tipo de alimentación y que refieren tanto a cuestiones de orden nutricional como medicinal, científico, moral, espiritual, político 
y económico, que comprenden diferentes formas de ver el mundo ${ }^{(29)}$.

Por otra parte, José, agricultor y ganadero de una localidad de Lérida, opina que no hay peligros en el alimento producido industrialmente:

No hay peligros en la producción industrial. Hoy en día no hay peligros en lo que comemos y bebemos. Hoy, todas las frutas que se recogen como mínimo han estado 10 días que no se han fumigado. Hoy todo está más controlado que en el pasado. (José, 49 años, Lérida)

Para Berta, ex trabajadora de una empresa química de Tarragona, la mejor manera de evitar los riesgos es, precisamente, utilizando bien las técnicas que el propio sistema capitalista proporciona para controlarlos. Berta entiende que estamos en un mundo mejor gracias a los avances de la industria y que apostar por este tipo de avances implica asumir unos riesgos que la propia industria se encarga de evitar:

Es que si queremos adelantos y evolucionar tenemos que asumir riesgos. Pero pienso que se ponen medidas de seguridad para que todo funcione bien y vayamos a mejor... (Berta, 63 años, Tarragona)

Por lo tanto, vemos que hay personas que aceptan y asumen determinados riesgos por el hecho de vivir en una sociedad industrializada. Con lo cual, podemos decir que cada cultura construye "riesgos asumibles" porque pueden comportar beneficios ${ }^{(30)}, \mathrm{y}$ lo que para unas sociedades es considerado objeto de temor o inseguridad no necesariamente lo es para otras ${ }^{(31)}$.

En este sentido, Juana, que trabaja como carnicera y vive en una localidad de la provincia de Barcelona, señala que los riesgos no son realidades objetivas, sino que dependen de cada sociedad, de las costumbres, maneras de ser y comer de cada momento y en cada territorio. Para Juana, los riesgos relacionados con los alimentos serían del mismo orden que otro tipo de riesgos propios de nuestra sociedad contemporánea, a la que cataloga de "obsesiva" y generadora de angustias y miedos innecesarios:

Depende de a lo que te hayas acostumbrado y de cada momento... Yo no soy nada obsesiva. Sé que los alimentos Ilevan conservantes, que no son naturales, pero, ¿por qué tengo que obsesionarme con ello? Creo que la persona no tiene que obsesionarse tanto con enfermedades, ni provocárselas, ni sentirse mal, ni sentirse intoxicada. No hace falta obsesionarse por si aquel pescado que estamos comiendo nos está pasando medio milígramo de mercurio [...] El exceso de información nos provoca angustias innecesarias. (Juana, 51 años, Barcelona)

De la misma manera, Gerard, el floricultor que anteriormente defendía la idea del alimento industrial como alimento inseguro y contaminado, en otro momento de la entrevista expresa sus dudas sobre los intereses económicos que puedan haber detrás de la construcción de los riesgos. Gerard comenta los peligros de los medios de comunicación en la difusión y reproducción de los intereses del mercado capitalista y en el control de la población a través de los discursos sobre los riesgos de los alimentos:

\begin{abstract}
A la gente, la televisión le come mucho el coco. En verdad, no sabes si un alimento lo han retirado del mercado porque era tóxico o porque a la multinacional no le interesaba y por eso lo ha dejado de fabricar. Igual lo han dejado de fabricar porque interesa vender otro producto para ganar más dinero y hacernos ver que ahora lo bueno para la salud está en otra cosa... (Gerard, 66 años, Tarragona)
\end{abstract}

\section{¿El riesgo está en el cómo? ¿Cómo se producen y controlan los alimentos?}

Muchos informantes sitúan la mirada en cómo están producidos los alimentos. 
Les preocupan los controles a los que están sometidos los alimentos, el ajuste entre el marco legal que los regula y los controles que realmente se practican, así como las técnicas de producción alimentaria que se aplican. En este sentido, regular y ejercer el control sanitario de los alimentos procesados para asegurar su inocuidad y calidad es una de las funciones y responsabilidades de los gobiernos.

No obstante, para Pedro, Raúl y Oriol, una cosa es la obligación legal y otra el cumplimiento real, ya que desconfían de los controles institucionales, sobre todo de aquellos que regulan los grandes monopolios agroalimentarios y farmacéuticos. Raúl, por su experiencia como veterinario y por las amistades que ha ido consolidando con ganaderos y otros veterinarios del sector, asegura que algunos productos químicos actualmente prohibidos continúan siendo utilizados de manera habitual y clandestina:

Los promotores de crecimiento, los clembuteroles, todas estas cosas que se ha demostrado que son cancerígenas. Estoy convencido que se siguen utilizando de forma clandestina. Tengo compañeros en el sector que lo pueden confirmar. (Raúl, 38 años, Tarragona)

Oriol, cocinero de un restaurante en Barcelona, también piensa que se continúan utilizando químicos prohibidos por ley. Según comenta, no se trata de rumorología, sino de un hecho que su tío, que se ha dedicado a la fabricación de piensos, ha podido constatar durante años:

Tengo un tío que se dedica a hacer pienso y lo ha visto hacer a otros. Para conseguir un contrato de venta de piensos en una granja, igual se le ofrecen más cosas. Una de ellas es esa vacuna que les ponen a las vacas cuatro o cinco días antes para que no orinen, para que pesen más. Esto está prohibidísimo pero se sigue haciendo. (Oriol, 41 años, Barcelona)
Por último, Pedro, miembro de una asociación ecologista, señala que más allá del uso clandestino de muchos productos químicos, tanto en la agricultura como en la ganadería persiste la problemática de cómo los propios laboratorios farmacéuticos manipulan las técnicas de análisis en función de sus intereses económicos:

Lo que pasa es que las analíticas cotidianas no van a buscar todos los tóxicos persistentes porque para eso hacen falta análisis más específicos y son mucho más caros. Además, la experiencia nuestra demuestra que cuando estas cosas se saben se tienden a esconder, porque con los nitratos se escondieron hasta que no hubo más remedio que sacarlo a la luz. (Pedro, 56 años, Baleares)

De hecho, algunos estudios ${ }^{(32)}$ muestran que el sistema español de seguridad alimentaria no es independiente a la hora de actuar sino que estaría sujeto a posibles intereses tanto políticos como económicos que pueden desvirtuar su objetivo principal.

Por otro lado, la desconfianza sobre la seguridad sanitaria de los alimentos puede recaer en los límites de las técnicas de control agroalimentario. Al respecto, Raúl, veterinario, señala que los análisis rutinarios parar detectar tóxicos no avanzan con la suficiente rapidez para captar los nuevos tóxicos que continuamente encuentran las investigaciones científicas. Por lo tanto, no siempre la legalidad se correspondería con la seguridad:

Es evidente que se realizan controles. Pero pasa lo mismo que con el dopaje: a medida que tú estableces una analítica rutinaria para detectar un producto, están saliendo de nuevos que no se detectan. Además, todo producto tiene un tiempo de supresión que si lo respetas, el nivel que pueda quedar residual es o indetectable a les analíticas o suficientemente bajo como para que la administración considere que es bastante bajo y no lo ilegalice. Es decir, se crean lo que 
se denomina niveles de seguridad y se crean porque aparece la presencia de una cosa nueva [...] Entonces, aquella lechuga contaminada que tengo a la nevera me la estoy comiendo yo. Y alguna cosa lleva que es legal, pero que probablemente también es nociva. (Raúl, 38 años, Tarragona)

También puede ser que la desconfianza se deposite en las biotecnologías aplicadas a la alimentación ${ }^{(6,7,9)}$ y se prefieran, como es el caso de Gerard, las técnicas tradicionales de producción que utilizaban sus antepasados:

Antes te contaban que hacían hormigueros, munts, o calentaban la tierra con brasas. Eso lo hacían porque a esa tierra le faltaba potasa o le faltaban unos microelementos que no tenía y entonces esa planta estiraba más de aquellos microelementos. Hoy no. Eso se lo das a la planta con la ferti-irrigación, con el abono... pero esto también tiene consecuencias sobre la salud y el medio ambiente. (Gerard, 66 años, Tarragona)

Por otro lado, la percepción del riesgo puede recaer en los propios límites de la legislación. En este caso, la mayoría de las personas entrevistadas señalan la heterogeneidad y diversidad de formas de regular la seguridad sanitaria de los alimentos según cada comunidad autónoma del Estado español y los diferentes países de los que se importan alimentos. Albert, ingeniero de una industria alimentaria, y Gerard, nos explican:

Se da la paradoja que hay productos que en España son permitidos y en otros países no, y otros productos que en España no están permitidos y en otros países sí. Con lo que te preguntas: ¿qué de malo tiene el acidulante E-330? (Albert, 50 años, Barcelona)

Hacen unas normas que no son iguales, por ejemplo, en la provincia de Almería, que en Murcia... Otro de los problemas es cuando nos llega algo de Marruecos, ya que si no hacen los controles necesarios y están autorizados estos productos tóxicos... (Gerard, 66 años, Tarragona)

Finalmente, casos como el de José, agricultor y ganadero de Lérida, y Albert, ingeniero industrial de Barcelona), ponen de manifiesto percepciones contrarias a las anteriores porque piensan que actualmente la legislación ha mejorado respecto al pasado y los controles alimentarios son más exhaustivos y eficaces:

Los controles son muy estrictos. Además, con la Declaración Única Agraria, saben las variedades que tengo en mi finca, dónde están situadas... lo saben todo. Tenemos inspecciones continuas. Estamos super-fiscalizados. Se tiene que tener fe, pero sí que funciona. (José, 49 años, Lérida)

El control existe y hay controles estrictos. Normalmente la normativa funciona [...] Tú no puedes hacer un producto que sea sanitariamente malo porque te lo retiran. (Albert, 50 años, Barcelona)

\section{¿El riesgo está en cuánto?: dosis, acumulación e incorporación}

Las percepciones alrededor del alimento contaminado también están marcadas por la relación que las personas establecen entre dosis-cantidad-acumulación y tiempo de exposición. Hay autores que afirman la tendencia a asumir las posibles cualidades negativas de un alimento sin tener en cuenta la cantidad ingerida dado que, a menudo, las personas clasifican algunos alimentos en tipologías como malo para comer sin tener en cuenta la cantidad ingerida ${ }^{(33,34)}$. No obstante, los casos de Luisa (técnica en alimentación), José (ganadero y agricultor) y Albert (ingeniero industrial) no se suman a esta observación. Para ellos, un alimento no se puede considerar bueno o malo para la salud sin tener en cuenta la cantidad y el tiempo de exposición a los elementos tóxicos o contaminantes: 
La producción donde se utilizan cada vez más E-s [aditivos alimentarios] $O$ conservantes, aromatizantes... creo que podría ser potencialmente una fuente de riesgo. Pero seguramente la exposición será baja. Hablamos de dosis muy bajas, pero comemos tres veces al día durante toda la vida. (Luisa, 35 años, Barcelona)

Si te has comido una pera que hace cuatro días estaba con piretrina, tu cuerpo no creo que lo pueda notar. Ahora, si tú te comes eso cada día... pienso que eso deja residuo y puede provocar, a la larga, problemas. (José, 49 años, Lérida)

Comerse un Donut no es perjudicial, comerse cada día una caja de seis es un problema. Pero también es un problema comerse cada día medio kilo de carne, aunque sea carne bio. (Albert, 50 años, Barcelona)

En este tipo de representaciones, el cuerpo es visto a menudo como un recipiente ${ }^{(24)}$ al que se le van sumando cantidades de sustancias contaminantes que dejan rastros y que con el tiempo pueden afectar negativamente la salud. De este modo:

...la idea de dosis no solo se aplica en función de una trayectoria pensada a largo plazo o diacrónica, sino que también se combina con otra de carácter sincrónico relacionada con la ingestión diaria de alimentos, donde la lógica sería que, ingiriendo pequeñas cantidades de contaminantes, la acumulación a largo plazo será menor. ${ }^{(24)}$

Otras veces, la dosis, la acumulación y el tiempo de exposición a un alimento se perciben a largo plazo. Entonces, se llega a la conclusión de que la aparición de una enfermedad será muy tardía y la preocupación por las consecuencias de esta resultan casi insignificantes $^{(24)}$. Es el caso de Juana, que trabaja en una carnicería de Barcelona, y piensa que "el día que me como un frankfurt, pues bueno... no pasa nada".
El cuerpo como recipiente vuelve a evidenciarse en el discurso de Juana, pero esta vez las consecuencias de la suma de contaminantes no son vistas como un probable desencadenante de enfermedad. Aquí el cuerpo es concebido como un recipiente capaz de adaptarse, asimilar, incluso neutralizar los posibles efectos negativos de los tóxicos y contaminantes:

Considero que el cuerpo se va adaptando a las porquerías que vamos asimilando. Entonces, bueno... [ríe] nos vamos... de generación en generación haciendo inmunes a ciertas cosas. Juana, 51 años, Barcelona)

El discurso de Juana, que concibe la capacidad de autoinmunizarse a los efectos de los peligros o contaminantes, nos pone de manifiesto cómo el discurso biomédico se incorpora en los discursos legos, reinterpretándose justo a la inversa de lo esperado por las políticas de salud pública, que pretenden concienciar y prevenir sobre los peligros de los contaminantes alimentarios. Además, se confirma la idea de que la modificación del saber a través de información no comporta necesariamente la modificación del comportamiento alimentario ${ }^{(31,35)}$.

Por otro lado, hemos observado que las experiencias de salud-enfermedad condicionan las maneras de entender y sentir el cuerpo en su relación con la contaminación, el riesgo alimentario y la salud. El caso de Marcos sería un ejemplo. Ex trabajador de la industria de cerámica, Marcos cambió por completo sus hábitos alimentarios y su filosofía de vida a raíz de la enfermedad de Crohn. La trayectoria de su enfermedad, así como sus consecuencias sociolaborales, han sido factores determinantes en el cambio progresivo de su visión respecto a los riesgos alimentarios dentro de un contexto de salud más global y holístico y, por extensión, en el cambio de su forma de entender el mundo, su corporalidad (física, psíquica y social) y sus formas de vida más vinculadas con el medio ambiente. Marcos y su pareja actualmente viven en una casa 
rural y se autoabastecen a partir de la agricultura ecológica.

\section{¿El riesgo está en quién, para quién o dónde se produce, distribuye o elabora un alimento?}

A muchos de los informantes les inquietan las vías de contacto con el tóxico o contaminante: el agua que riega un huerto, el aire que envuelve un producto o la tierra en la que se cultiva. Este puede ser un motivo que lleva a muchas personas a comprar alimentos a quienes les tienen confianza: "a mi vecina que tiene un huerto y sé que no pone insecticidas"; "al verdulero de los miércoles en el mercadillo porque lo conozco y todo lo lleva bueno", etc. En estos casos, lo que se escoge no es el alimento en sí, sino dónde y quién lo ha producido por la confianza que, en calidad de cercanía y proximidad, genera su producción y distribución.

Además, la percepción de inseguridad sobre un producto no solo proviene de la cercanía o lejanía en la que la industria sitúa su producción, sino del lugar dónde esté situada la propia industria: en lugares que implican una percepción previa sobre confianza o desconfianza. En este sentido, resulta paradójico que haya personas que prefieran productos de origen industrial antes que otros de origen natural cuya procedencia sea un país poco industrializado. Jaime, agricultor y miembro de una cooperativa agrícola de Tarragona señala:

Desconfío de los productos que nos llegan, sobre todo, de países con un nivel de vida más bajo que el nuestro. Yo siempre digo que si comiésemos productos de proximidad y de temporada seguramente evitaríamos muchos peligros. (Jaime, 60 años, Tarragona)

Estos discursos ponen en evidencia cómo la otredad, la distancia física y social y categorías como conocido o desconocido, cercano o lejano participan de y en la construcción social de los riesgos alimentarios.
Por último, la percepción sobre el riesgo también puede variar en función de para quién se elaboren o preparen los alimentos. Un ejemplo sería Laura, madre de una niña, bióloga y propietaria de un comercio de alimentación ecológica que explica su sensibilización con respecto a este tipo de alimentos cuando se quedó embarazada y, posteriormente, cuando se responsabilizó de la alimentación de su hija. Su concepción sobre el cuerpo embarazado justificó la incorporación de productos ecológicos, considerados por ella como más naturales, saludables y con menos contaminantes. Empezó leyendo artículos científicos sobre el tema, intercambiando información con otras madres y finalmente reconfigurando sus maneras de pensar y hacer la alimentación dentro de esquemas sobre salud y cuerpo más interconectados, en los que el cuerpo no se ve únicamente como un recipiente que acumula contaminantes o tóxicos, sino como un ente activo en relación con el medioambiente y capaz de evitar y modificar los riesgos que la rodean porque, según ella:

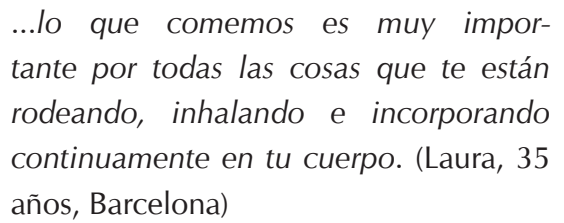

\section{DISCUSIONES Y CONCLUSIONES FINALES}

Nuestro estudio ha permitido analizar las percepciones sociales sobre el riesgo alimentario en el conjunto de diversas narrativas en el ámbito de Cataluña, que inciden en qué alimentos son percibidos como peligrosos y focalizando la atención en cómo, cuándo, dónde, por qué y de qué manera está concebido este riesgo a partir de las propias experiencias de los informantes.

En términos generales, se ha demostrado que los procesos sociales de construcción del riesgo alimentario son multifactoriales, diversos y conjugan elementos a menudo 
paradójicos y que contradicen los conocimientos expertos -basados primordialmente en los factores biotecnológicos y macroeconómicos de la alimentación-y legos, en los que participan muchas otras variables de carácter histórico, individual y cultural-simbólicas.

Así pues, las percepciones sobre el alimento contaminado transcienden al propio alimento, para construirse, también, a partir del contexto que lo rodea. Un alimento en sí mismo puede considerarse seguro o inseguro pero, sobre todo, son las formas, los lugares, las personas que lo produen o para quienes se produce, distribuye, prepara o consume el alimento, lo que verdaderamente le confiere el significado sobre su seguridad o inseguridad. De este modo, la percepción sobre el riesgo alimentario no solo es construida conforme a la visión biomédica de un cuerpo físico, químico o biológico que se introduce en el interior de un alimento (como los aditivos, los pesticidas o las hormonas, por ejemplo), sino también en función de su corporalidad individual, sociocultural y política.

En este sentido, el estudio señala que el debate sobre las percepciones del riesgo alimentario no se puede reducir solamente a una cuestión de estar o no a favor del progreso o de la industrialización ni, por extensión, al debate dicotómico y unívoco que muchas veces se hace alrededor del alimento bueno o malo, peligroso o seguro, contaminado o puro, artificial o natural, industrializado o ecológico, ya que se han puesto de manifiesto testimonios que perciben precisamente como alimento seguro el que ha sido procesado industrialmente. Asimismo, hay informantes que ponen en duda la categoría natural, incluso algunos que cuestionan la seguridad de los alimentos ecológicos. A la vez, algunas personas conciben el riesgo alimentario como fruto de intereses económicos y políticos, como costumbres y hábitos incorporados socioculturalmente, incluso como una obsesión contemporánea.

Además, hemos visto que la percepción sobre el riesgo también puede recaer en cómo están producidos los alimentos, más concretamente, en el ajuste entre el marco legal que los regula y el que realmente se aplica y las técnicas de producción y control que se utilizan. No obstante, de la misma manera que se defienden estas ideas, encontramos discursos totalmente confiados con relación a las formas de control actuales y, por lo tanto, con percepciones que apuntan a que no existe inseguridad sanitaria en los alimentos y, por extensión, tampoco riesgo alimentario.

Por otra parte, algunos resultados evidencian la construcción social y simbólica de la confianza-desconfianza sobre los alimentos a partir de categorías que tienen que ver con la otredad, lo conocido y lo desconocido, así como la distancia física y social respecto del lugar donde se produce, distribuye o prepara un alimento (quién, dónde, para quién). Del mismo modo, se han encontrado relaciones entre cuerpo, riesgo y alimentación en las que la dosis-acumulación-tiempo de exposición a un alimento contaminado (cuánto de riesgo) nos refieren a una perspectiva en la que el cuerpo es visto como un recipiente ${ }^{(24)}$ al que se le van sumando cantidades de sustancias contaminantes que se acumulan y que, con el tiempo, podrían generar enfermedades. Sin embargo, otras veces las consecuencias de la suma de contaminantes no son vistas ni sentidas como una probable causa de enfermedad porque el cuerpo es pensado en su capacidad de adaptarse, asimilar, incluso neutralizar estos posibles efectos negativos.

Estos resultados ponen en evidencia dos cuestiones que, aunque no han sido abordadas directamente en el estudio, se desprenden del análisis e interpretación de las narrativas. Por un lado, el hecho de que se acepten y asuman determinados riesgos por vivir en una sociedad industrializada es indicativo de que cada cultura construye ciertos riesgos asumibles porque pueden comportar beneficios ${ }^{(30)}$, y lo que para unas sociedades es considerado objeto de temor o inseguridad no necesariamente lo es para otras ${ }^{(31)}$. Por otro lado, el cuerpo individual, social y político ${ }^{(17)}$ está emergiendo como centro a partir del cual se construyen las percepciones sobre el riesgo alimentario contemporáneo, de manera que este (el cuerpo) no solo se asocia a un objeto pasivo (un recipiente) al que van 
a parar y acumularse los contaminantes, sino también a un sujeto activo del proceso de construcción de lo que se considera seguro o inseguro en términos de salud alimentaria.

Finalmente, toda esta complejidad inherente a los procesos de construcción del riesgo alimentario nos conduce inevitablemente a reflexionar sobre la necesidad de (re)pensar y aplicar enfoques teóricos y metodológicos que la tengan en cuenta. En este sentido, son necesarias perspectivas y métodos que superen los reduccionismos y que relacionen adecuadamente los vínculos inseparables que existen entre cuerpo, alimentación, riesgo y salud. Asimismo, precisamos políticas inclusivas que recuperen la riqueza del discurso lego y su construcción histórica, económica y sociopolítica; y que no consideren a los sujetos únicamente como agentes pasivos y receptivos acríticos de la información, sino como actores activos -con capacidad individual, social y política- del proceso de (re) construcción de un mundo natural y social mejor y más seguro.

\section{AGRADECIMIENTOS}

Los resultados presentados en este texto forman parte del proyecto interdisciplinario "Cuerpos tóxicos y etnoepidemiología sociocultural de la contaminación interna por compuestos tóxicos persistentes (CTP) en España", adscrito al Departamento de Antropología Social de la Universidad de Barcelona, que contó con el apoyo y la financiación del Programa Nacional de Proyectos de Investigación Fundamental del Ministerio de Economía y Competitividad (CSO 2010/18661). Los autores agradecen la colaboración de los miembros del proyecto y expresan su especial gratitud a todos los informantes que participaron en la investigación por compartir su tiempo y sus experiencias.

\section{REFERENCIAS BIBLIOGRÁFICAS}

1. Agencia Catalana de Seguridad Alimentaria. Barómetro de la seguridad alimentaria en Cataluña. Barcelona: Departament de Salut de la Generalitat de Catalunya; 2012.

2. Apfelbaum M. (dir.). Risques et peurs alimentaires. Paris: Odil Jacob; 1998.

3. Bredahl L. Consumer's cognitions with regard to genetically modified foods: Results of a qualitative study in four countries. Appetite. 1999;33:343-360.

4. Chateauraynaud F, Torny D. Surveiller et contenir: un monde peuplé de prions. En: Chateauraynaud F, Torny D, (eds.). Les sombres precurseurs: 
Une sociologie pragmatique de I'alerte et du risque. Paris: Éditions de l’Ehess; 1999.

5. Latouch K, Rainelli P, Vermesch D. Food safety issues and the BSE scare: some lessons from the French case. Food Policy. 1999;23(5):347-356.

6. Butz P, Needs EC, Baron A, Bayer O, Geisel B, Oltersdorf U, Tauscher C. Consumer, attitudes to high pressure foodprocessing. Journal of Food, Agriculture and Environment. 2003;1(1):30-34.

7. Gaskell G, Ten Eyck T, Jackson J, Veltri G. Imagining nanotechnology: cultural support for technological innovation in Europe and the United States. Public Understanding of Science. 2005;14(1):81-90.

8. European Commission. Eurobarometer 66 . Public opinion in the European Union. [Internet]. 2006 [citado 10 feb 2016]. Disponible en: http:// tinyurl.com/y4f4b2a.

9. Cáceres J, Espeitx E. Les noves tecnologies aplicades a I'alimentació: factors d'acceptació i rebuig a Catalunya [Internet]. Barcelona: Agència Catalana de Seguretat Alimentaria, 2012 [citado 30 mar 2016]. Disponible en: http://tinyurl.com/hkjddho.

10. Fundación Vasca para la Seguridad Agroalimentaria. Estudio de percepción en seguridad alimentaria en la comunidad autónoma del País Vasco [Internet]. País Vasco: Gobierno Vasco; 2012 [citado 20 feb 2016]. Disponible en: http:// tinyurl.com/jnvfyoa.

11. Slorach SA. Enfoques Integrados para la gestión de la inocuidad de los alimentos a lo largo de toda la cadena alimentaria. En: Foro Mundial FAO/OMS de las Autoridades de Reglamentación sobre Inocuidad de los Alimentos; Marrakech, Marruecos, 28-30 de enero de 2002 [Internet]. GF 01/10 [citado 27 sep 2016]. Disponible en: http:// tinyurl.com/zdjpv6b.

12. Organización Mundial de la Salud. Temas de Salud: Inocuidad de los Alimentos. [Internet] 2016 [citado 26 sep 2016]. Disponible en: http://tinyurl. com/jys5hh4.

13. Douglas M. Pureza y peligro: Un análisis de los conceptos de contaminación y tabú. Madrid: Siglo XXI Editores; 1973.

\section{Lupton D. Risk. London: Routledge; 1999.}

15. Oaks L, Harthon BH. Health and the social and cutural construction of risk. In: Harthon $\mathrm{BH}$, Oaks L, (eds). Risk, culture, and health inequality: shifting perceptions of danger and blame. Westport CT: Praeger; 2003.
16. Jensen $M$, Blok $A$. Pesticides in the risk society: The view from everyday life. Current Sociology. 2008;56(5):757-778.

17. Sheper-Hughes $N$, Lock $M$. The mindful body: A prolegomenon to future work in medical anthropology. Medical Anthropology Quarterly. 1987;1(1):6-41.

18. Confederación de Comercio de Catalunya [Internet]. 2006 [citado 23 ene 2015]. Disponible en: http://www.confecom.cat.

19. Foucault M. Vigilar y castigar. Madrid: Siglo XXI Editores; 1992.

20. Glaser BG, Strauss AL. The discovery of grounded theory: strategies for qualitative research. Chicago: Aldine Publishing Company; 1967.

21. Strauss AL, Corbin J. Basics of qualitative research: grounded theory procedures and techniques. Newbury Park: Sage Publishing; 1990.

22. Medina FX. El color de la tradición: Color y construcción del pasado en la publicidad alimentaria. En: Barusi A, Medina FX, Colesanti G. El color en la alimentación mediterránea: Elementos sensoriales y culturales de la nutrición. Barcelona: Institut Català de la Mediterrània; 1998.

23. Fischler C. El (h)omnívoro. Barcelona: Anagrama; 1995.

24. Guidonet A. ¿Miedo a comer?: Crisis alimentaria en contextos de abundancia. Barcelona: Icaria y Observatorio de la Alimentación; 2010.

25. Rozin P, Fischler C, Shields-Argelès C. European and American perspectives on the meaning of natural. Appetite. 2012;59(2):448-455.

26. Rozin P. La préférence pour le natural. In: Fischler C, Masson E. Manger: Français, Européens et Américains face à I'alimentation. Paris: Odile Jacob; 2008.

27. Baranski M, Srednicka D, Volakakis N, Seal C, Sanderson GB, Steward R, Bebrook C, Biavati B, Markellou E, Giotis C, Gromadzka J, RembiaIkowska E, Skwarlo K, Tahvonen R, Janovská D, Niggli $U$, Nicot $P$, Leifert $C$. Higher antioxidant and lower cadmium concentrations and lower incidence of pesticide residues in organically grown crops: A systematic literature review and meta-analysis. British Journal of Nutrition. 2014;112(5):794-811.

28. Mulet JM. Los productos naturales ¡vaya timo! Pamplona: Editorial Laetoli; 2011. 
29. Begueria A. Un equilibrio imperfecto: Alimentación ecológica, cuerpo y toxicidad. Barcelona: Editorial UOC; 2016.

30. Peretti-Watel P. Sociologie du risque. Paris: Armand Colin; 2000.

31. Contreras J, Gracia M. Alimentación y cultura: Perspectivas antropológicas. Barcelona: Ariel; 2005.

32. PonceG, MuñozE. La percepción de los actores de la seguridad alimentaria. Arbor. 2005;715:393-402. doi: 10.3989/arbor.2005.i715.420.
33. Fischler C. Raison et déraison dans les perceptions des risques alimentaires. Cahiers de Nutrition et de Diététique. 1998;33(5):297-301.

34. Fischler C. Food selection and risk perception. In: Anderson HB, Chiva M. Food selection: From genes to culture. Levallois-Perret: Danone Institute; 2002.

35. Zafra E. Comer en la escuela: el modelo de educación alimentaria en el comedor escolar. Revista Trabajo Social y Salud. 2005;51:361-382.

\section{FORMA DE CITAR}

Zafra Aparici E, Muñoz García A, Larrea-Killinger C. ¿Sabemos lo que comemos?: Percepciones sobre el riesgo alimentario en Cataluña, España. Salud Colectiva. 2016;12(4):505-518. doi: 10.18294/sc.2016.932

Recibido: 5 de abril de 2016 | Versión final: 27 de septiembre de 2016 | Aprobado: 7 de octubre de 2016

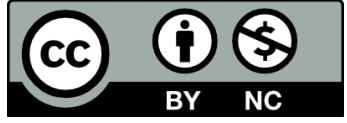

Este obra está bajo una licencia de Creative Commons Reconocimiento-NoComercial 4.0 Internacional. Reconocimiento - Permite copiar, distribuir y comunicar públicamente la obra. A cambio, se debe reconocer y citar al autor original. No Comercial - Esta obra no puede ser utilizada con finalidades comerciales, a menos que se obtenga el permiso.

http://dx.doi.org/10.18294/sc.2016.932 